\title{
MIXED WEAK TYPE ESTIMATES: EXAMPLES AND COUNTEREXAMPLES RELATED TO A PROBLEM OF E. SAWYER
}

\author{
S.OMBROSI AND C. PÉREZ
}

\section{INTRODUCTION AND MAIN RESULtS}

In this work we study mixed weighted weak-type inequalities of the form

$$
u v\left(\left\{x \in \mathbb{R}^{n}: \frac{|T(f v)(x)|}{v(x)}>t\right\}\right) \leq \frac{C}{t} \int_{\mathbb{R}^{n}}|f(x)| M u(x) v(x) d x,
$$

where to fix ideas, the operator $T$ is either the Hardy-Littlewood maximal operator or any Calderón-Zygmund Operator. Versions of these type of inequalities were studied by Sawyer in [Sa] motivated by the work of Muckenhoupt and Wheeden [MW] (see also the works [AM] and [MOS]).

E. Sawyer proved that inequality (1.1) holds in $\mathbb{R}$ when $T=M$ is the Hardy-Littlewood maximal operator if the weights $u$ and $v$ belong to the class $A_{1}$. Although this result can be seen as a very delicate extension of the classical weak type $(1,1)$ estimate, the reason why E. Sawyer was interested on inequality it is due to the following interesting observation. Indeed, this inequality yields a new proof of Muckenhoupt's classical theorem assuming that the $A_{p}$ weights can be factored (P. Jones's theorem), namely if $w \in A_{p}$ then $w=w_{1} w_{2}^{1-p}$ for some $w_{1}, w_{2} \in A_{1}$. In fact, we have that the operator $f \rightarrow \frac{M(f v)}{v}$ is bounded on $L^{\infty}(u v)$ and the same operator satisfies (1.1). Hence by interpolation we recover Muckenhoupt's theorem.

In the same paper, Sawyer conjectured that if $T$ is instead the Hilbert transform the inequality also holds with the same hypotheses on the weights $u$ and $v$. That conjecture was proved in [CMP2]. In fact, it is proved in this paper that the inequality (1.1) holds for both the Hardy-Littlewood maximal operator and for any Calderón-Zygmund Operator in any dimension $n$ if either the weights $u$ and $v$ belong to $A_{1}$ or $u$ belongs to $A_{1}$ and $u v \in A_{\infty}$. The authors conjectured that their results may hold with weaker hypotheses. To be more precise they propose that inequality (1.1) is true if $u \in A_{1}$ and $v \in A_{\infty}$. The method of proof is quite different from that in

The first author is grateful to the Department of Mathematical Analysis of the Universidad de Sevilla for the hospitality, and he is partially supported by SGCyT,UNS and a fellowship from Junta of Andalucía, the second author is partially supported by DGICYT Grant PB980106. 
[Sa] (also from $[\mathrm{MW}]$ ) and it is based on certain ideas from extrapolation that goes back to the work of Rubio de Francia (see [CMP2] and also the review [CMP3]).

In this work we generalize the extrapolation result in [CMP3]), for a more general class of weights (see Theorem 1.1 below). This method of extrapolation is flexible enough that can be applied the result goes beyond the classical linear operators. Indeed, it can be applied to square functions, vector valued operators as well and multilinear singular integral operators. See Section 2 for some of these applications.

When $T$ is the Hardy-Littlewood maximal operator we can think that this type of inequalities can be considered like a generalization of the classical Fefferman-Stein inequality. However, in Section 3, we will see that the inequality (1.1) in general even taking weights $v \in R H_{\infty} \subset A_{\infty}$ does not hold.

The best way to state the extrapolation theorem is without considering operators. In fact the result is a property of families of functions. Hereafter, $\mathcal{F}$ will denote a family of ordered pairs of non-negative, measurable functions $(f, g)$. Also we are going to assume that this family $\mathcal{F}$ of functions, satisfies the following property: for some $p_{0}, 0<p_{0}<\infty$, and every $w \in A_{\infty}$,

$$
\int_{\mathbb{R}^{n}} f(x)^{p_{0}} w(x) d x \leq C \int_{\mathbb{R}^{n}} g(x)^{p_{0}} w(x) d x,
$$

for all $(f, g) \in \mathcal{F}$ such that the left-hand side is finite, and where $C$ depends only on the $A_{\infty}$ constant of $w$. By the main theorem in [CMP1], this assumption turns out to be equivalent to that for every $p, 0<p<\infty$, and every $w \in A_{\infty}$,

$$
\int_{\mathbb{R}^{n}} f(x)^{p} w(x) d x \leq C \int_{\mathbb{R}^{n}} g(x)^{p} w(x) d x,
$$

for all $(f, g) \in \mathcal{F}$ such that the left-hand side is finite, and where $C$ depends only on the $A_{\infty}$ constant of $w$. See [CMP1], [CGMP] and the survey paper [CMP3] for more information and applications.

It is also interesting that both 1.2 and 1.3 are equivalent to the following vector-valued version: for all $0<p, q<\infty$ and for all $w \in A_{\infty}$ we have

$$
\left\|\left(\sum_{j}\left(f_{j}\right)^{q}\right)^{\frac{1}{q}}\right\|_{L^{p}(w)} \leq C\left\|\left(\sum_{j}\left(g_{j}\right)^{q}\right)^{\frac{1}{q}}\right\|_{L^{p}(w)},
$$

for any $\left\{\left(f_{j}, g_{j}\right)\right\}_{j} \subset \mathcal{F}$, where these estimates hold whenever the left-hand sides are finite.

Next theorem improves the corresponding Theorem from [CMP2]. Indeed, observe that weights of the form $v(x)=|x|^{-n r}$ for any $r>0$ are 
included in the hypothesis of the Theorem but not in the corresponding Theorem from [CMP2] when $r \geq 1$, namely the singular case.

Theorem 1.1. Let $\mathcal{F}$ be a family of functions satisfying (1.2) and let $\theta \geq 1$. Suppose that $u \in A_{1}$ and that $v$ is a weight such that for some $\delta>0$, $v^{\delta} \in A_{\infty}$.

Then, there is a constant $C$

$$
\left\|\frac{f}{v^{\theta}}\right\|_{L^{1 / \theta, \infty}(u v)} \leq C\left\|\frac{g}{v^{\theta}}\right\|_{L^{1 / \theta, \infty}(u v)}, \quad(f, g) \in \mathcal{F} .
$$

Similarly, there is the following vector-valued extension: for any $0<$ $p, q<\infty$

$$
\left\|\frac{\left.\sum_{j}\left(f_{j}\right)^{q}\right)^{\frac{1}{q}}}{v^{\theta}}\right\|_{L^{1 / \theta, \infty}(u v)} \leq C\left\|\frac{\left.\sum_{j}\left(g_{j}\right)^{q}\right)^{\frac{1}{q}}}{v^{\theta}}\right\|_{L^{1 / \theta, \infty}(u v)},
$$

for any $\left\{\left(f_{j}, g_{j}\right)\right\}_{j} \subset \mathcal{F}$.

The proof of (1.6) is immediate since we can extrapolate using as initial hypothesis (1.4) applying (1.5).

Corollary 1.2. Let $\mathcal{F}, u$ and $\theta \geq 1$ as in the Theorem. Suppose now that $v_{i}, i=1, \cdots, m$, are weights such that for some $\delta_{i}>0, v_{i}^{\delta_{i}} \in A_{\infty}$, $i=1, \cdots, m$.

Then, if we denote $v=\prod_{i=1}^{m} v_{i}$

$$
\left\|\frac{f}{v^{\theta}}\right\|_{L^{1 / \theta, \infty}(u v)} \leq C\left\|\frac{g}{v^{\theta}}\right\|_{L^{1 / \theta, \infty}(u v)}, \quad(f, g) \in \mathcal{F} .
$$

and similarly for all $0<p, q<\infty$,

$$
\left\|\frac{\left.\sum_{j}\left(f_{j}\right)^{q}\right)^{\frac{1}{q}}}{v^{\theta}}\right\|_{L^{1 / \theta, \infty}(u v)} \leq C\left\|\frac{\left.\sum_{j}\left(g_{j}\right)^{q}\right)^{\frac{1}{q}}}{v^{\theta}}\right\|_{L^{1 / \theta, \infty}(u v)},
$$

for any $\left\{\left(f_{j}, g_{j}\right)\right\}_{j} \subset \mathcal{F}$.

The proof reduces to the Theorem by choosing $\delta>0$ small enough such that $v^{\delta}=\prod_{i=1}^{m} v_{i}^{\delta} \in A_{\infty}$ which follows by convexity since $v_{i}^{\delta_{i}} \in A_{\infty}, i=$ $1, \cdots, m$.

To apply the extrapolation theorem above to some of the classical operators we need a mixed weak type estimate for the Hardy-Littlewood maximal operator. In fact by that Theorem we just need the dyadic version.

The next Theorem was obtained in dimension one by Andersen and Muckenhoupt in $[\mathrm{AM}]$ and by Martín-Reyes, Ortega Salvador and Sarrión Gavián [MOS] in higher dimensions. In each case the proof follows as a consequence 
of a more general result with the additional hypothesis that $u \in A_{1}$. However, for the sake of completeness we will give an independent and direct proof with no condition on the weight $u$.

Theorem 1.3. Let $u \geq 0$ and $v(x)=|x|^{-n r}$ for some $r>1$. Then there is a constant $C$ such that for all $t>0$,

$$
u v\left(\left\{x \in \mathbb{R}^{n}: \frac{M(f v)(x)}{v(x)}>t\right\}\right) \leq \frac{C}{t} \int_{\mathbb{R}^{n}}|f(x)| M u(x) v(x) d x .
$$

Remark 1.4. We remark that, in general, the case $r=1$ is false for the previous theorem even in the case $u=1$, see [AM]. However, we already mentioned that weights of the form $v(x)=|x|^{-n r}, r>0$ are included in the extrapolation Theorem 1.1.

Acknowledgement. The authors are grateful to F. J. Martín-Reyes and P. Ortega-Salvador to point out reference [MOS].

\section{Some APPLICATIONS}

In this section we show the flexibility of the method by giving two applications.

2.1. The vector-valued case. Let $T$ be any singular integral operator with standard kernel and let $M$ is the Hardy-Littlewood maximal function. We are going to show that starting from the following inequality due to Coifman [Coi]: for $0<p<\infty$ and $w \in A_{\infty}$,

$$
\int_{\mathbb{R}^{n}}|T f(x)|^{p} w(x) d x \leq C \int_{\mathbb{R}^{n}} M f(x)^{p} w(x) d x,
$$

which combined with the extrapolation Theorem 1.1 together with Theorem 1.3 yields the following corollary.

Corollary 2.1. Let $u \in A_{1}$ and $v(x)=|x|^{-n r}$ for some $r>1$. Also let $1<q<\infty$. Then, there is a constant $C$ such that for all $t>0$,

$$
\begin{aligned}
& u v\left(\left\{x \in \mathbb{R}^{n}: \frac{\left(\sum_{j} M\left(f_{j} v\right)(x)^{q}\right)^{\frac{1}{q}}}{v(x)}>t\right\}\right) \leq \frac{C}{t} \int_{\mathbb{R}^{n}}\left(\sum_{j}\left|f_{j}(x)\right|^{q}\right)^{\frac{1}{q}} u(x) v(x) d x, \\
& u v\left(\left\{x \in \mathbb{R}^{n}: \frac{\left(\sum_{j}\left|T\left(f_{j} v\right)(x)\right|^{q}\right)^{\frac{1}{q}}}{v(x)}>t\right\}\right) \leq \frac{C}{t} \int_{\mathbb{R}^{n}}\left(\sum_{j}\left|f_{j}(x)\right|^{q}\right)^{\frac{1}{q}} u(x) v(x) d x .
\end{aligned}
$$

Observe that in particular we have the following scalar version:

$$
u v\left(\left\{x \in \mathbb{R}^{n}: \frac{|T(f v)(x)|}{v(x)}>t\right\}\right) \leq \frac{C}{t} \int_{\mathbb{R}^{n}}|f(x)| u(x) v(x) d x .
$$


This scalar version was proved in [MOS].

To proof of the second inequality of the Corollary, follows from the first one by applying inequality (1.6) in Theorem 1.1 with initial hypothesis (2.1):

$$
\begin{aligned}
& \sup _{t>0} \operatorname{tuv}\left(\left\{x \in \mathbb{R}^{n}: \frac{\left(\sum_{j}\left|T\left(f_{j}\right)(x)\right|^{q}\right)^{\frac{1}{q}}}{v(x)}>t\right\}\right) \leq \\
& C \sup _{t>0} \operatorname{tuv}\left(\left\{x \in \mathbb{R}^{n}: \frac{\left(\sum_{j} M\left(f_{j}\right)(x)^{q}\right)^{\frac{1}{q}}}{v(x)}>t\right\}\right) .
\end{aligned}
$$

To prove the first inequality in Corollary 2.1 we first note that in [CGMP] was shown for $1<q<\infty$ and for all $0<p<\infty$ and $w \in A_{\infty}$,

$$
\left\|\left(\sum_{j}\left(M\left(f_{j}\right)\right)^{q}\right)^{\frac{1}{q}}\right\|_{L^{p}(w)} \leq C\left\|M\left(\left(\sum_{j}\left|f_{j}\right|^{q}\right)^{\frac{1}{q}}\right)\right\|_{L^{p}(w)} .
$$

To conclude we apply Theorem 1.1 combined with Theorem 1.3.

2.2. Multilinear Calderón-Zygmund operators: We now apply our main results to multilinear Calderón-Zygmund operator. We follow here the theory developed by Grafakos and Torres in [GT1], that is, $T$ is an $m$-linear operator such that $T: L^{q_{1}} \times \cdots \times L^{q_{m}} \longrightarrow L^{q}$, where $1<q_{1}, \ldots, q_{m}<\infty$, $0<q<\infty$ and

$$
\frac{1}{q}=\frac{1}{q_{1}}+\cdots+\frac{1}{q_{m}} .
$$

The operator $T$ is associated with a Calderón-Zygmund kernel $K$ in the usual way:

$T\left(f_{1}, \ldots, f_{m}\right)(x)=\int_{\mathbb{R}^{n}} \ldots \int_{\mathbb{R}^{n}} K\left(x, y_{1}, \ldots, y_{m}\right) f_{1}\left(y_{1}\right) \ldots f_{m}\left(y_{m}\right) d y_{1} \ldots d y_{m}$,

whenever $f_{1}, \ldots, f_{m}$ are in $C_{0}^{\infty}$ and $x \notin \bigcap_{j=1}^{m} \operatorname{supp} f_{j}$. We assume that $K$ satisfies the appropriate decay and smoothness conditions (see [GT1, GT2] for complete details). Such an operator $T$ is bounded on any other product of Lebesgue spaces with exponents $1<q_{1}, \ldots, q_{m}<\infty, 0<q<\infty$ satisfying (2.2). Further, it also satisfies weak endpoint estimates when some of the $q_{i}$ 's are equal to one. There are also weighted norm inequalities for multi-linear Calderón-Zygmund operators; these were first proved in [GT2] using a good- $\lambda$ inequality, and later in [PT] using the sharp maximal function. They showed that for $0<p<\infty$ and for all $w \in A_{\infty}$,

$$
\left\|T\left(f_{1}, \ldots, f_{m}\right)\right\|_{L^{p}(w)} \leq C\left\|\prod_{j=1}^{m} M f_{j}\right\|_{L^{p}(w)} .
$$


Beginning with these inequalities, we can apply Theorem 1.1 to the family $\mathcal{F}\left(T\left(f_{1}, \ldots, f_{m}\right), \prod_{j=1}^{m} M f_{j}\right)$. Hence, if $u \in A_{1}$ and $v(x)=|x|^{-n r}$ for some $r>1$.

$$
\left\|\frac{\left|T\left(f_{1}, \ldots, f_{m}\right)\right|}{v^{m}}\right\|_{L^{1 / m, \infty}(u v)} \leq C\left\|\frac{\prod_{j=1}^{m} M f_{j}}{v^{m}}\right\|_{L^{1 / m, \infty}(u v)}
$$

Corollary 2.2. Let $T$ be a multilinear Calderón-Zygmund operator as above. Let $u \in A_{1}$ and $v(x)=|x|^{-n r}$ for some $r>1$. Then

$$
\left\|\frac{\left|T\left(f_{1}, \ldots, f_{m}\right)\right|}{v^{m}}\right\|_{L^{1 / m, \infty}(u v)} \leq C \prod_{j=1}^{m} \int_{\mathbb{R}^{n}}\left|f_{j}\right| u d x
$$

To prove this corollary we will use the following version of the generalized Holder's inequality: for $1 \leq q_{1}, \ldots, q_{m}<\infty$ with

$$
\frac{1}{q_{1}}+\cdots+\frac{1}{q_{m}}=\frac{1}{q}
$$

there is a constant $C$ such that

$$
\left\|\prod_{j=1}^{m} h_{j}\right\|_{L^{q, \infty}(w)} \leq C \prod_{j=1}^{m}\left\|h_{j}\right\|_{L^{q_{j}, \infty}(w)} .
$$

The proof of this inequality follows in a similar way that the proof of the classic generalized Holder's inequality in $L^{p}$ Theory.

Now, if we combine this together with (2.3) we have

$$
\left\|\frac{\left|T\left(f_{1}, \ldots, f_{m}\right)\right|}{v^{m}}\right\|_{L^{1 / m, \infty}(u v)} \leq C \prod_{j=1}^{m}\left\|\frac{M f_{j}}{v}\right\|_{L^{1, \infty}(u v)},
$$

So from this and (1.3) we conclude the proof of the corollary.

\section{COUNTEREXAMPLES}

We consider that it is an interesting fact that we can obtain in Theorem 1.3

$$
u v\left(\left\{x \in \mathbb{R}^{n}: \frac{M(f v)(x)}{v(x)}>t\right\}\right) \leq \frac{C}{t} \int_{\mathbb{R}^{n}}|f(x)| M u(x) v(x) d x \quad u \geq 0
$$

for $v(x)=|x|^{-n r}, r>1$. On the other hand from the works [Sa] and [CMP3] we know that the same inequality holds if $u \in A_{1}$ and $v \in A_{1}$ or $u v \in A_{\infty}$. A natural question is whether we could prove inequality (3.1) for these class of weights $v$ improving the classical Fefferman-Stein inequality. However, we will show in next example that this is false in general.

Before giving this example, we observe that if $u \in A_{1}$ and $v$ belongs to $R H_{\infty}$ the inequality (3.1) holds. This fact follows since $u v \in A_{\infty}$, and by Theorem 1.4 in [CMP2]. 
Example 3.1. Let $v(x)=\sum_{k \in Z}|x-k| \chi_{I_{k}}(x)$, where $I_{k}$ denote the interval $|x-k| \leq 1 / 2$, it is not difficult to see that $v \in R H_{\infty}$. If we choose $u(x)=$ $\sum_{\substack{k \in N \\ k>10}} \frac{k}{\log (k)} \chi_{J_{k}}(x)$ with $J_{k}=\left[k+\frac{1}{4 k}, k+\frac{1}{k}\right]$, and $f(x)=\chi_{[-1,1]}(x)$ then it does not exist a finite constant $C$ such that the inequality

$$
u v(\{x: M f(x)>v(x)\}) \leq C \int|f| M^{2} u
$$

holds.

We will the following observation:

In $\mathbb{R}^{n}$, there is a geometric constant such that

$$
M^{2} w(x) \approx M_{L \log L} w(x)
$$

where

$$
M_{L \log L} f(x)=\sup _{Q \ni x}\|f\|_{L \log L, Q}
$$

and

$$
\|f\|_{L \log L, Q}=\inf \left\{\lambda>0: \frac{1}{|Q|} \int_{Q} \Phi\left(\frac{|f|}{\lambda}\right) d x \leq 1\right\} .
$$

with $\Phi(t)=t \log (e+t)$, see $[\mathrm{PW}]$ or $[\mathrm{G}]$. Now, it is a computation to see that if $x \in[-1,1], M^{2} u(x) \approx M_{L \log L} u(x) \leq C$ so the right hand side of (3.2) is finite, and however the left hand side of (3.2) is infinite. We will see that. For $|x|>2$ we have that $M f(x) \geq \frac{1}{|x|}$ and if $x \in J_{k} \subset I_{k}$ for $k>10 \frac{1}{|x|}>\frac{1}{2 k}$, then it is easy to see that $\left(k+\frac{1}{4 k}, k+\frac{1}{2 k}\right) \subset\left\{x \in J_{k}\right.$ : $M f(x)>v(x)\}$ and therefore we obtain that

$$
\begin{aligned}
u v(\{x: M f(x)>v(x)\}) & >\sum_{\substack{k \in N \\
k>10}} \frac{k}{\log (k)} \int_{k+\frac{1}{4 k}}^{k+\frac{1}{2 k}}(x-k) d x> \\
& >\sum_{\substack{k \in N \\
k>10}} \frac{1}{8 k \log (k)}=\infty .
\end{aligned}
$$

\section{Proof of Theorem 1.1}

The following Lemmas will be useful:

Lemma 4.1. If $u \in A_{1}, w \in A_{1}$, then there exists $0<\epsilon_{0}<1$ depending only on $[u]_{A_{1}}$ such that $u w^{\epsilon} \in A_{1}$ for all $0<\epsilon<\epsilon_{0}$.

Proof. Since $u \in A_{1}, u \in R H_{s_{0}}$ for some $s_{0}>1$ depending on $[u]_{A_{1}}$. Let $\epsilon_{0}=1 / s_{0}{ }^{\prime}$ and $0<\epsilon<\epsilon_{0}$. This implies that $u \in R H_{s}$ with $s=(1 / \epsilon)^{\prime}$.

Then since $u, v \in A_{1}$, for any cube $Q$ and almost every $x \in Q$,

$$
\frac{1}{|Q|} \int_{Q} u(y) w(y)^{\epsilon} d y \leq\left(\frac{1}{|Q|} \int_{Q} u(y)^{s} d y\right)^{1 / s}\left(\frac{1}{|Q|} \int_{Q} w(y) d y\right)^{1 / s^{\prime}}
$$




$$
\leq \frac{[u]_{R H_{s}}}{|Q|} \int_{Q} u(y) d y\left(\frac{1}{|Q|} \int_{Q} w(y) d y\right)^{1 / s^{\prime}} \leq[u]_{R H_{s}}[u]_{A_{1}}[w]_{A_{1}}^{\epsilon} u(x) w(x)^{\epsilon} .
$$

Hence $u w^{\epsilon} \in A_{1}$ with $\left[u w^{\epsilon}\right]_{A_{1}} \leq[u]_{R H_{s}}[u]_{A_{1}}[w]_{A_{1}}^{\epsilon}$.

Also we need the following version of the Marcinkiewicz interpolation theorem in the scale of Lorentz spaces. In fact we need a version of this theorem with precise constants. The proof can be found in [CMP2].

Proposition 4.2. Given $p_{0}, 1<p_{0}<\infty$, let $T$ be a sublinear operator such that

$$
\|T f\|_{L^{p_{0}, \infty}} \leq C_{0}\|f\|_{L^{p_{0}, 1}} \quad \text { and } \quad\|T f\|_{L^{\infty}} \leq C_{1}\|f\|_{L^{\infty}} .
$$

Then for all $p_{0}<p<\infty$,

$$
\|T f\|_{L^{p, 1}} \leq 2^{1 / p}\left(C_{0}\left(1 / p_{0}-1 / p\right)^{-1}+C_{1}\right)\|f\|_{L^{p, 1}} .
$$

Fix $u \in A_{1}$ and $v$ such that $v^{\delta} \in A_{\infty}$ for some $\delta>0$. Then by the factorization theorem $v^{\delta}=v_{1} v_{2}$ for some $v_{1} \in A_{1}$ and $v_{2} \in R H_{\infty}$. Define the operator $S_{\lambda}$ by

$$
S_{\lambda} f(x)=\frac{M\left(f u v_{1}^{1 / \lambda \delta}\right)}{u v_{1}^{1 / \lambda \delta}}
$$

for some large enough constant $\lambda>1$ that will be chosen soon.

By Lemma 4.1, there exists $0<\epsilon_{0}<1$ (that depends only on $[u]_{A_{1}}$ ) such that $u w^{\epsilon} \in A_{1}$ for all $w \in A_{1}$ and $0<\epsilon<\epsilon_{0}$.

Hence we choose $\lambda>\frac{1}{\delta \epsilon_{0}}$ such that $u v_{1}^{1 / \lambda \delta} \in A_{1}$. Hence, $S_{\lambda}$ is bounded on $L^{\infty}(u v)$ with constant $C_{1}=[u]_{A_{1}}$. We will now show that for some larger $\lambda, S_{\lambda}$ is bounded on $L^{m}(u v)$. Observe that

$$
\int_{\mathbb{R}^{n}} S f(x)^{\lambda} u(x) v(x) d x=\int_{\mathbb{R}^{n}} M\left(f u v_{1}^{1 / \lambda \delta}\right)(x)^{\lambda} u(x)^{1-\lambda} v_{2}(x)^{1 / \delta} d x .
$$

Since $v_{2}=\tilde{v}_{2}^{1-t}$ for some $\tilde{v}_{2} \in A_{1}$ and $t>1$. Hence,

$$
u^{1-\lambda} v_{2}^{1 / \delta}=u^{1-\lambda} \tilde{v}_{2}^{\frac{1-t}{\delta}}=\left(u \tilde{v}_{2}^{\frac{t-1}{\delta(\lambda-1)}}\right)^{1-\lambda} .
$$

By Lemma 4.1 there exists $\lambda$ even bigger if necessary $\left(\lambda>1+\frac{t-1}{\delta \epsilon_{0}}\right)$ such that $u \tilde{v}_{2}^{\frac{t-1}{\delta(\lambda-1)}} \in A_{1}$ and hence $u^{1-\lambda} v_{2}^{1 / \delta} \in A_{\lambda}$. By Muckenhoupt's theorem, $M$ is bounded on $L^{\lambda}\left(u^{1-\lambda} v_{2}^{1 / \delta}\right)$ and therefore $S$ is bounded on $L^{\lambda}(u v)$ with some constant $C_{0}$. Observe that $\lambda$ depends upon the $A_{1}$ constant of $u$. We fix one of these $\lambda$ from now on.

Thus by Proposition 4.2 above we have that $S$ is bounded on $L^{q, 1}(u v)$, $q>\lambda$. Hence,

$$
\|S f\|_{L^{q, 1}(u v)} \leq 2^{1 / q}\left(C_{0}(1 / \lambda-1 / q)^{-1}+C_{1}\right)\|f\|_{L^{q, 1}(u v)} .
$$


Thus, for all $q \geq 2 \lambda$ we have that $\|S f\|_{L^{q, 1}(u v)} \leq K_{0}\|f\|_{L^{q, 1}(u v)}$ with $K_{0}=$ $4 \lambda\left(C_{0}+C_{1}\right)$. We emphasize that the constant $K_{0}$ is valid for every $q \geq 2 \lambda$.

Fix $(f, g) \in \mathcal{F}$ such that the left-hand side of (1.5) is finite. We let $\theta<r<\theta(2 \lambda)^{\prime}$ that is going to be chosen soon. Now, by the duality of $L^{r, \infty}$ and $L^{r^{\prime}, 1}$

$\left\|f v^{-\theta}\right\|_{L^{1 / \theta, \infty}(u v)}^{\frac{1}{r}}=\left\|\left(f v^{-\theta}\right)^{\frac{1}{r}}\right\|_{L^{r / \theta, \infty}(u v)}=\sup \int_{\mathbb{R}^{n}} f(x)^{\frac{1}{r}} h(x) u(x) v(x)^{1-\theta / r} d x$,

where the supremum is taken over all non-negative $h \in L^{\left(\frac{r}{\theta}\right)^{\prime}, 1}(u v)$ with $\|h\|_{L^{\left(\frac{r}{\theta}\right)^{\prime}, 1}(u v)}=1$. Fix such a function $h$. We are going to build a larger function $\mathcal{R} h$ using the Rubio de Francia's method such $\mathcal{R} h u v^{1-\theta / r} \in A_{\infty}$. Hence we will use the hypothesis (1.3) with $p=\theta / r$ (recall that is equivalent to (1.2)) with the weight $\mathcal{R} h u v^{1-\theta / r} \in A_{\infty}$

We let $r$ such that $\left(\frac{r}{\theta}\right)^{\prime}>2 \lambda$ and hence $S_{\left(\frac{r}{\theta}\right)^{\prime}}$ is bounded on $L^{\left(\frac{r}{\theta}\right)^{\prime}, 1}(u v)$ with constant bounded by $K_{0}$. Now apply the Rubio de Francia algorithm (see $[\mathrm{GCRdF}]$ ) to define the operator $\mathcal{R}$ on $h \in L^{\left(\frac{r}{\theta}\right)^{\prime}, 1}(u v), h \geq 0$, by

$$
\mathcal{R} h(x)=\sum_{j=0}^{\infty} \frac{S_{\left(\frac{r}{\theta}\right)^{j}}^{j} h(x)}{2^{j} K_{0}^{j}},
$$

Recall that the operator $S_{\left(\frac{r}{\theta}\right)^{\prime}}$ is defined by

$$
S_{\left(\frac{r}{\theta}\right)^{\prime}} f(x)=\frac{M\left(f u v_{1}^{1 /\left(\frac{r}{\theta}\right)^{\prime} \delta}\right)}{u v_{1}^{1 /\left(\frac{r}{\theta}\right)^{\prime} \delta}} .
$$

Recall that by the choice of $r u v_{1}^{1 /\left(\frac{r}{\theta}\right)^{\prime} \delta} \in A_{1}$.

It follows immediately from this definition that:

(a) $h(x) \leq \mathcal{R} h(x)$

(b) $\|\mathcal{R} h\|_{L^{\left(\frac{r}{\theta}\right)^{\prime}, 1}(u v)} \leq 2\|h\|_{L^{\left(\frac{r}{\theta}\right)^{\prime}, 1}(u v)} ;$

(c) $S_{\left(\frac{r}{\theta}\right)^{\prime}}(\mathcal{R} h)(x) \leq 2 K_{0} \mathcal{R} h(x)$.

In particular, it follows from $(c)$ and the definition of $S$ that $\mathcal{R} h u v_{1}^{1 /\left(\frac{r}{\theta}\right)^{\prime} \delta} \in$ $A_{1}$ and therefore $\mathcal{R} h u v^{1 /\left(\frac{r}{\theta}\right)^{\prime}}=\mathcal{R} h u v_{1}^{1 / \delta\left(\frac{r}{\theta}\right)^{\prime}} v_{2}^{1 / \delta\left(\frac{r}{\theta}\right)^{\prime}} \in A_{\infty}$.

To apply the hypothesis (1.3) we must first check that the left-hand side is finite, but this follows at once from Hölder's inequality and (b):

$$
\begin{aligned}
\int_{\mathbb{R}^{n}} f(x)^{\frac{1}{r}} \mathcal{R} h(x) u(x) v(x)^{1-\frac{\theta}{r}} d x & \leq\left\|\left(f v^{-\theta}\right)^{\frac{1}{r}}\right\|_{L^{r / \theta, \infty}(u v)}\|\mathcal{R} h\|_{L^{(r / \theta)^{\prime}, 1}(u v)} \\
& \leq 2\left\|f v^{-\theta}\right\|_{L^{1 / \theta, \infty}(u v)}^{\frac{1}{r}}\|h\|_{L^{\left(\frac{r}{\theta}\right)^{\prime}, 1}(u v)}<\infty .
\end{aligned}
$$

Thus since $\mathcal{R} h u v^{1 /\left(\frac{r}{\theta}\right)^{\prime}} \in A_{\infty}$ by $(1.3)$

$$
\int_{\mathbb{R}^{n}} f(x)^{\frac{1}{r}} h(x) u(x) v(x)^{1-\frac{\theta}{r}} d x \leq \int_{\mathbb{R}^{n}} f(x)^{\frac{1}{r}} \mathcal{R} h(x) u(x) v(x)^{1-\frac{\theta}{r}} d x
$$




$$
\begin{aligned}
& \leq C \int_{\mathbb{R}^{n}} g(x)^{\frac{1}{r}} \mathcal{R} h(x) u(x) v(x)^{1-\frac{\theta}{r}} d x \\
& \leq C\left\|\left(g v^{-\theta}\right)^{\frac{1}{r}}\right\|_{L^{r / \theta, \infty}(u v)}\|\mathcal{R} h\|_{L^{\left(\frac{r}{\theta}\right)^{\prime}, 1}(u v)} \\
& \leq 2 C\left\|g v^{-\theta}\right\|_{L^{1 / \theta, \infty}(u v)}^{\frac{1}{r}} .
\end{aligned}
$$

Since $C$ is independent of $h$, inequality (1.5) follows finishing the proof of the theorem.

\section{Proof of Theorem 1.3}

5.1. Proof of (1.7). The following lemma is important in the proof.

Lemma 5.1. Let $f$ be a positive and locally integrable function. Then for $r>1$ there exists a positive real number a depending on $f$ and $\lambda$ such that the inequality

$$
\left(\int_{|y| \leq a^{\frac{1}{r-1}}} f(y) d y\right) a^{n}=\lambda
$$

holds.

Proof. Consider the function

$$
g(a)=\left(\int_{|y| \leq a^{\frac{1}{r-1}}} f(y) d y\right) a^{n}, \text { for } a \geq 0,
$$

then by the hypothesis we have that $g$ is a continuous and non decreasing function. Furthermore, $g(0)=0$, and $g(+\infty)=+\infty$, and therefore, by the mean value theorem there exists $a$ such that satisfies the conditions of lemma.

By simplicity we denote $g=f v$, furthermore by homogeneity we can assume that $\lambda=1$. We denote $G_{k}=\left\{2^{k}<|x| \leq 2^{k+1}\right\}, I_{k}=\left\{2^{k-1}<|x| \leq 2^{k+2}\right\}$, $L_{k}=\left\{2^{k+2}<|x|\right\}, C_{k}=\left\{|x| \leq 2^{k-1}\right\}$.

It will be enough to prove the following estimates

$$
\begin{aligned}
& \sum_{k \in Z} \frac{u(x)}{|x|^{n r}}\left\{x \in G_{k}: M g \chi_{I_{k}}(x)>\frac{1}{|x|^{n r}}\right\} \leq C_{r, n} \int g M u, \\
& \sum_{k \in Z} \frac{u(x)}{|x|^{n r}}\left\{x \in G_{k}: M g \chi_{L_{k}}(x)>\frac{1}{|x|^{n r}}\right\} \leq C_{r, n} \int g M u, \\
& \sum_{k \in Z} \frac{u(x)}{|x|^{n r}}\left\{x \in G_{k}: M g \chi_{C_{k}}(x)>\frac{1}{|x|^{n r}}\right\} \leq C_{r, n} \int g M u,
\end{aligned}
$$


Taking into account that in $G_{k}, v(x)=\frac{1}{|x|^{n r}} \sim 2^{-k n r}$, using the $(1,1)$ weak type inequality of $M$ with respect to the pair of weights $(u, M u)$ and since the subsets $I_{k}$ are overlapping at most three times we obtain (5.1).

By the inequality (5.2) we will estimate $M g \chi_{L_{k}}(x)$. Observe that if $x$ belongs to $G_{k}$ and $y \in L_{k}=\left\{2^{k+2}<|y|\right\}$, and if $|y-x| \leq \rho$, we have that $\frac{|y|}{2} \leq \rho$,

$$
\frac{1}{\rho^{n}} \int_{|y-x| \leq \rho} g(y) \chi_{L_{k}}(y) d y \leq C_{n} \int_{2^{k+2}<|y|} \frac{g(y)}{|y|^{n}} d y \leq C_{n} \int_{|x|<|y|} \frac{g(y)}{|y|^{n}} d y .
$$

If we denote $F(x)=\int_{|x|<|y|} \frac{g(y)}{|y|^{n}} d y$ the left hand side in (5.2) is bounded by

$$
\begin{gathered}
\sum_{k \in Z} 2^{-k r n} u\left\{x \in \mathbb{R}^{n}: F(x)>C 2^{-k n r}\right\} \approx \int_{0}^{\infty} t u\left\{x \in \mathbb{R}^{n}: F(x)>t\right\} \frac{d t}{t} \\
=\int_{\mathbb{R}^{n}} F(x) u(x) d x=\int_{\mathbb{R}^{n}} \int_{|x|<|y|} \frac{g(y)}{|y|^{n}} d y u(x) d x \\
=\int_{\mathbb{R}^{n}} g(y) \frac{1}{|y|^{n}} \int_{|x|<|y|} u(x) d x d y \leq C \int_{\mathbb{R}^{n}} g(y) M u(y) d y .
\end{gathered}
$$

Now we will estimate $M g \chi_{C_{k}}(x)$ for $x \in G_{k}$. For $x \in G_{k}$, si $y \in C_{k}$, $2|y|<|x|$ and since $M g \chi_{C_{k}}(x) \leq c_{n} \frac{1}{|x|^{n}} \int_{C_{k}} g(y) d y$, we obtain

$$
M g \chi_{C_{k}}(x) \leq \frac{C}{|x|^{n}} \int_{C_{k}} g \leq \frac{C}{|x|^{n}} \int_{|y| \leq \frac{|x|}{2}} g,
$$

Thus, since the subsets $G_{k}$ are disjoints, the left hand side in (5.3) is bounded by

$$
\frac{u(x)}{|x|^{n r}}\left\{x \in \mathbb{R}^{n}: \frac{C}{|x|^{n}} \int_{|y| \leq \frac{|x|}{2}} g>\frac{1}{|x|^{n r}}\right\} .
$$

Now, if $a$ denotes the positive real number that appears in Lemma 5.1 (i.e., $a$ satisfies that $1=\left(\int_{|y| \leq a^{\frac{1}{r-1}}} g\right) a^{n}$, we express the last integral in the following way:

$$
\begin{aligned}
& \frac{u(x)}{|x|^{n r}}\left(\left\{x: \frac{C}{|x|^{n}} \int_{|y| \leq \frac{|x|}{2}} g>\frac{1}{|x|^{n r}}\right\}\right)=\frac{u(x)}{|x|^{n r}}\left(\left\{|x| \leq a^{\frac{1}{r-1}}: \frac{C}{|x|^{n}} \int_{|y| \leq \frac{|x|}{2}} g>\frac{1}{|x|^{n r}}\right\}\right)+ \\
& \quad+\sum_{k=0}^{\infty} \frac{u(x)}{|x|^{n r}}\left(\left\{x \in 2^{k} a^{\frac{1}{r-1}}<|x| \leq 2^{k+1} a^{\frac{1}{r-1}}: \frac{C}{|x|^{n}} \int_{|y| \leq \frac{|x|}{2}} g>\frac{1}{|x|^{n r}}\right\}\right)
\end{aligned}
$$

If $|x| \leq a^{\frac{1}{r-1}}$, since $|y| \leq \frac{|x|}{2}$ we have that $|y| \leq a^{\frac{1}{r-1}}$, thus the set

$$
\left\{|x| \leq a^{\frac{1}{r-1}}: \frac{C}{|x|^{n}} \int_{|y| \leq \frac{|x|}{2}} g>\frac{1}{|x|^{n r}}\right\} \subset\left\{|x| \leq a^{\frac{1}{r-1}}:|x|^{n(r-1)}>C\left(\int_{|y| \leq a^{\frac{1}{r-1}}} g\right)^{-1}\right\} .
$$


Taking into account the last inclusion and since $\left(\int_{|y| \leq a^{\frac{1}{r-1}}} g\right)^{-1}=a^{n}$, the first summand in the second term in (5.4) is bounded by

$$
\frac{u(x)}{|x|^{n r}}\left(\left\{|x|^{r-1}>C a\right\}\right)=\frac{u(x)}{|x|^{n r}}\left(\left\{|x|>c a^{r^{\prime}-1}\right\}\right) .
$$

Using again Lemma 5.1, the last term can been estimated by

$$
\begin{gathered}
\int_{|x|>C a^{r^{\prime}-1}} \frac{u(x)}{|x|^{n r}} d x \leq C \sum_{k=1}^{\infty} \frac{1}{\left(2^{k} a^{r^{\prime}-1}\right)^{n r}} \int_{c 2^{k-1} a^{r^{\prime}-1} \leq|x|<c 2^{k} a^{r^{\prime}-1}} u(x) d x \leq \\
\leq C \sum_{k=1}^{\infty} \frac{1}{2^{k(r-1) n}} \frac{1}{a^{n}} \frac{1}{\left(c 2^{k} a^{r^{\prime}-1}\right)^{n}} \int_{|x| \leq c 2^{k} a^{r^{\prime}-1}} u(x) d x \\
=C \sum_{k=1}^{\infty} \frac{1}{2^{k(r-1) n}} \int_{|y| \leq a^{r^{\prime}-1}} f(y) d y \frac{1}{\left(c 2^{k} a^{r^{\prime}-1}\right)^{n}} \int_{|x| \leq c 2^{k} a^{r^{\prime}-1}} u(x) d x
\end{gathered}
$$

And this term is bounded by

$$
\leq C \sum_{k=1}^{\infty} \frac{1}{2^{k(r-1) n}} \int_{|y| \leq a^{r^{\prime}-1}} g(y) M w(y) d y \leq C \int g M u
$$

To finish, we must estimate the series in (5.4). It is clear that sum is bounded by

$\sum_{k=0}^{\infty} \frac{u(x)}{|x|^{n r}}\left(\left\{x \in 2^{k} a^{r^{\prime}-1}<|x| \leq 2^{k+1} a^{r^{\prime}-1}\right\}\right) \leq C \sum_{k=0}^{\infty} \frac{1}{\left(2^{k} a^{r^{\prime}-1}\right)^{n r}} \int_{2^{k-1} a^{r^{\prime}-1} \leq|x|<2^{k} a^{r^{\prime}-1}} u d x$

and arguing as before we conclude the proof of (5.3).

Remark 5.2. We observe that the proof only uses the following conditions for a sublinear operator $T$ : a) $T$ is of weak type $(1,1)$ with respect to the pair of weight $(u, M u)$ and b) $T$ is a convolution type operator such that the associate kernel satisfies the usual standard condition:

$$
|K(x)| \leq \frac{c}{|x|^{n}}
$$

In particular if $u \in A_{1}$, this observation can be applied to the usual CalderónZygmund Singular Integral Operators and moreover to the Strongly Singular Integral Operators (see $[\mathrm{Ch}]$ and $[\mathrm{F}]$ ). 


\section{REFERENCES}

[AM] K. Andersen, B. Muckenhoupt Weighted weak type Hardy inequalities with applications to Hilbert transforms and maximal functions, Studia Math. 72 (1982), no. $1,9-26$.

[Ch] S. Chanillo, Weighted norm inequalities for strongly singular convolution operators. Trans. Am. Math. Soc. V 281, (1984).

[Coi] R.R. Coifman, Distribution function inequalities for singular integrals, Proc. Acad. Sci. U.S.A. 69 (1972), 2838-2839.

[CMP1] D. Cruz-Uribe, J.M. Martell and C. Pérez, Extrapolation results for $A_{\infty}$ weights and applications, J. Funct. Anal. 213 (2004) 412-439.

[CMP2] D. Cruz-Uribe, J.M. Martell and C. Pérez, Weighted weak-type inequalities and a conjecture of Sawyer, International Mathematics Research Notices, 30 2005, 1849-1871.

[CMP3] D. Cruz-Uribe, J.M. Martell y C. Pérez, Extensions of Rubio de Francia's extrapolation theorem, Collectanea Mathematica 57 (2006) 195-231.

[CGMP] G.P. Curbera, J. García-Cuerva, J.M. Martell and C. Pérez, Extrapolation with Weights, Rearrangement Invariant Function Spaces, Modular inequalities and applications to Singular Integrals, Advances in Mathematics, 203 (2006) 256318.

[F] C. Fefferman. Inequalities for strongly singular convolution operator, Acta Math. 124 (1970) 9-36.

[GCRdF] J. García-Cuerva and J.L. Rubio de Francia, Weighted Norm Inequalities and Related Topics, North Holland Math. Studies 116, North Holland, Amsterdam, 1985.

[G] L. Grafakos, Classical and Modern Fourier Analysis, Pearson Education, New Jersey, 2004.

[GT1] L. Grafakos and R. Torres, Multilinear Calderón-Zygmund theory, Adv. in Math. 165 (2002), 124-164.

[GT2] L. Grafakos and R. Torres, Maximal operator and weighted norm inequalities for multilinear singular integrals, Indiana Univ. Math. J. 51 (2002), no. 5, 12611276.

[MOS] F. J. Martín-Reyes, P. Ortega Salvador, M. D. Sarrión Gavilán, Boundedness of operators of Hardy type in $\Lambda^{p, q}$ spaces and weighted mixed inequalities for singular integral operators, Proc. Roy. Soc. Edinburgh Sect. A 127 (1997), no. 1, 157-170.

[MW] B. Muckenhoupt and R. Wheeden, Some weighted weak-type inequalities for the Hardy-Littlewood maximal function and the Hilbert transform, Indiana Math. J. 26 (1977), 801-816.

[PT] C. Pérez and R. Torres, Sharp maximal function estimates for multilinear singular integrals, to appear in Contemporary Mathematics, Proceedings to the Mount Holyoke Summer Research School in Harmonic Analysis.

[PW] C. Pérez and R. Wheeden, Uncertaitny principle estimates for vector fields, Journal of Functional Analysis 181 (2001), 146-188.

[Sa] E.T. Sawyer, A weighted weak type inequality for the maximal function, Proc. Amer. Math. Soc. 93 (1985), 610-614.

Departmento de Matemáticas, Universidad Nacional del Sur, Bahía Blanca

E-mail address: sombrosi@uns.edu.ar

Departamento de Análisis Matemático, Facultad de Matemáticas, UniVersidad de Sevilla, 41080 Sevilla, Spain

E-mail address: carlosperez@us.es 